\title{
Reconstruction of the Mandible with a Poly(D,L-lactide) Scaffold, Autogenous Corticocancellous Bone Graft, and Autogenous Platelet-Rich Plasma: An Animal Experiment*
}

\author{
JEROEN P.M. FENNIS, D.D.S., M.D., PAUL J.W. STOELINGA, D.D.S., M.D., Ph.D., \\ MATTHIAS A.W. MERKX, D.D.S., M.D., Ph.D., and JOHN A. JANSEN, D.D.S., Ph.D.
}

\begin{abstract}
An animal study is presented, evaluating a method of mandibular reconstruction using a poly(D,Llactide) (PDLLA) scaffold. Six goats underwent a continuity resection of the mandibular angle. The defect was bridged with a preshaped PDLLA scaffold, filled with an autogenous particulate bone graft from the anterior iliac crest, and fixed with two preshaped titanium plates. To accelerate bone healing, autogenous platelet-rich plasma was mixed with the particulate bone graft. All goats had an uneventful healing. The osteosynthesis system withstood immediate loading for a period of 6 weeks until sacrifice. The particulate bone grafts within the PDLLA scaffold, which appeared to be narrowed, showed considerable resorption and replacement by fibrous tissue. In all goats, however, callus formation along the reconstructed segment was seen, providing bony continuity and maintaining the original contour of the reconstructed segment. Thus, the technique used may provide an alternative for reconstruction with revascularized composite flaps with less associated donor site morbidity.
\end{abstract}

\section{INTRODUCTION}

$\mathbf{C}$ UnRent ReConstructive techniques for continuity defects of the mandible encompass the use of revascularized free flaps, free nonvascularized bone grafts, and alloplastic materials. It still remains a challenge to restore the three-dimensional shape of the hard and soft tissues and, at the same time, to allow for functional rehabilitation by means of implant-supported prostheses. Although revascularized composite flaps may be regarded as the "gold standard," they are associated with considerable donor site morbidity. ${ }^{1,2}$ The present study forms part of an ongoing project in which it is attempted to achieve reconstruction of the mandible that comes as close as pos- sible to the original contour of the resected part of the mandible. For this reason, it was thought mandatory to use autogenous or alloplastic scaffolds, filled with an autogenous bone graft, to mimic the skeletal anatomy of the original lower jaw as closely as possible. Previous studies have shown that the experimental animal model used to create and subsequently bridge a mandibular continuity defect, using two $2.3-\mathrm{mm}$ plates, turned out to be adequate. A study in which the resected cortical bone segment was reimplanted as an autogenous cortical scaffold filled with a particulate cancellous iliac crest bone graft, with or without platelet-rich plasma (PRP), provided insight concerning the contribution of PRP to the healing of bone grafts. Radiological and histological evaluation

Department of Oral and Maxillofacial Surgery, Rijnstate Hospital, Arnhem, The Netherlands.

*This study was carried out at the Department of Oral of Maxillofacial Surgery and at the Department of Biomaterials, University Medical Centre Nijmegen, Nijmegen, The Netherlands. 
showed that the addition of PRP enhanced bone-healing considerably. ${ }^{3,4}$ In the PRP group, extensive bone union, more callus formation along the grafted segment, a larger amount of vital bone surface, and a higher number of capillaries within the cancellous bone graft were seen, as compared with the non-PRP group. It was also found that in animals treated with PRP, there were fewer tendencies to form a fibrous capsule around the grafted segment. The differences between the PRP and non-PRP groups became apparent after 6 weeks and maturation and remodeling of the bone graft appeared to continue between 6 and 12 weeks. In another animal experiment, the scaffolds were irradiated extracorporeally before reimplantation and filled with a particulate iliac crest bone graft mixed with PRP, with the idea of eliminating any eukaryotic cells in the cortical bone tray. In the clinical situation this should prevent tumor recurrence. ${ }^{5}$ Bone healing, however, was not as successful as in the group with the nonirradiated scaffolds. The specimens showed more fibrous encapsulation of the grafts and lower capillary counts within the grafted segments. ${ }^{6}$

An alternative to an autologous irradiated cortical bone scaffold would be an alloplastic scaffold. ${ }^{7-12}$ The animal experiment described here tests the versatility of a technique in which the resected part of the mandible is replaced by a resorbable poly(D,L-lactide) (PDLLA) scaffold, filled with a particulate cancellous bone graft from the anterior iliac crest and mixed with PRP. The hypothesis is that these grafts will heal, providing bone continuity with the grafted bone, within a period of 6 weeks.

\section{MATERIALS AND METHODS}

Successful previous and comparable experiments ${ }^{3}$ caused the Animal Ethics Committee of the University of Nijmegen (Nijmegen, The Netherlands) to grant permission for the current study, for which six goats were used.

Before surgery blood samples of the goats were taken to ensure that the animals were caprine or thritis-encephalitis/caseous lymphadenitis (CAE/CL) free. The animals were housed in a stable. National guidelines for the care and use of laboratory animals were observed. The operations were performed under general anesthesia. The anesthesia was induced by an intravenous injection of pentobarbital and maintained with 2-3\% isoflurane through a constant-volume ventilator, administered through an endotracheal tube. The goats were connected to a heart monitor.

To reduce the risk of perioperative infection, the goats were treated with three doses of ampicillin; the second and third doses were depots:

During the operation: $15 \%$ ampicillin, $3 \mathrm{~mL} / 50 \mathrm{~kg}$, subcutaneous
Day 1 postoperation: ampicillin, $7.5 \mathrm{~mL} / 50 \mathrm{~kg}$, subcutaneous

Day 3 postoperation: ampicillin, $7.5 \mathrm{~mL} / 50 \mathrm{~kg}$, subcutaneous

Before surgery, the skin at the operation sites was shaved and disinfected with povidone-iodine solution. An incision of $5 \mathrm{~cm}$ was made on top of the anterior iliac crest. Subsequently subperiosteal elevation was carried out on the lateral aspect of the crest. Approximately $5 \mathrm{~cm}^{3}$ of corticocancellous bone was harvested by means of a trephine. ${ }^{13}$ The wound was closed in layers, using resorbable sutures (Vicryl; Ethicon, Somerville, NJ). The harvested bone was ground in a bone mill (Stryker Leibinger Micro Implants, Freiburg, Germany). Consequently the particulate bone graft consisted of cancellous and cortical bone.

A continuity resection on the right side of the mandible, measuring $4 \mathrm{~cm}$ at the lower border and 1.5 $\mathrm{cm}$ in the retromolar area, was performed, including the angle of the mandible, through a submandibular approach. The approach was subperiosteal; on the buccal aspect the periosteum was excised, whereas on the lingual side the periosteum was left intact in order not to damage the lingual nerve. Before resection, two preshaped osteosynthesis plates (Martin Medizintechnik, Tüttlingen, Germany) were adapted, using proprietary bending tools, in order to maintain the correct position of the mandible. The osteosynthesis plates were created by computer-aided design to fit the contour of a goat mandible and automatically transformed into the computer-aided manufacturing process (CAD-CAM). This guaranteed that the shape of the plates would fit an average adult goat mandible, which obviated the need for major bending of the plates. The plates had a thickness

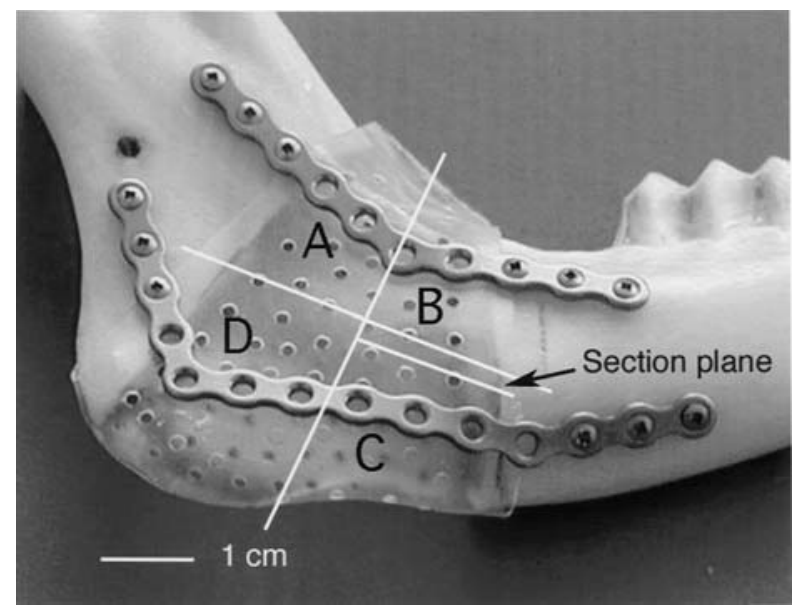

FIG. 1. PDLLA scaffold made to fit the average goat mandible. Fixation of the stumps is with a preshaped $2.3-\mathrm{mm}$ plate system. Only part $\mathrm{C}$ was used for histological sections, that is, $3 \mathrm{~mm}$ below the cut between $\mathrm{B}$ and $\mathrm{C}$ (section plane). 
Table 1. Parameters and Scoring System Used for RAdiographic Evaluation

\begin{tabular}{|c|c|}
\hline Parameter & Score \\
\hline $\begin{array}{l}\text { Bone union } \\
\text { (continuity of bone } \\
\text { within defect) }\end{array}$ & $\begin{aligned} 0= & \text { Discontinuity of the bone } \\
1= & \text { Partial obliteration } \\
2= & >90 \% \text { obliteration } \\
3= & \text { increased radio-opacity } \\
& \text { compared with untreated } \\
& \text { mandible }\end{aligned}$ \\
\hline $\begin{array}{l}\text { Callus formation } \\
\text { (at anterior and posterior } \\
\text { osteotomy sites) }\end{array}$ & $\begin{array}{l}0=\text { None } \\
1=\text { Present }\end{array}$ \\
\hline $\begin{array}{l}\text { Central resorption } \\
\text { (internal resorption) }\end{array}$ & $\begin{array}{l}0=\text { None } \\
1=\text { Present }\end{array}$ \\
\hline $\begin{array}{l}\text { External resorption } \\
\text { (external resorption } \\
\text { at lower border) }\end{array}$ & $\begin{array}{l}0=\text { None } \\
1=\text { Present }\end{array}$ \\
\hline
\end{tabular}

of $1.5 \mathrm{~mm}$ and a width of $5.4 \mathrm{~mm}$. They were manufactured from titanium alloy (DIN 17851.1990-11-00 titanium alloys; chemical composition, ASTM F136-98). The center drive (Martin Medizintechnik) screws had a diameter of $2.3 \mathrm{~mm}$ and a pitch of $1.25 \mathrm{~mm}$. The screws were manufactured from alloyed titanium (DIN 17851.1990-11-00 titanium alloys; chemical composition, ASTM F136-98).

The neurovascular bundle entering the mandible was ligated in order to control bleeding after resection. The segment was then resected, using a fissure burr no. 2. A series of poly-D,L-lactide (PDLLA [Martin Medizintechnik]/Resomer R208 [Boehringer Ingelheim, Ingelheim am Rhein, Germany]) trays was fabricated, by means of heat and vacuum treatment, to fit the average mandibular angle of a goat (Fig. 1). This preshaped PDLLA tray was then adapted to the goat recipient site and filled with an autogenous particulate bone graft taken from the anterior iliac crest mixed with PRP. The particulate bone graft was completely enveloped by the perforated PDLLA tray.

For this reason, $250 \mathrm{~cm}^{3}$ of blood was drawn 2 days before surgery. This blood was treated by centrifugation at the Sanquin Bloodbank Nijmegen in various cycles. ${ }^{14}$
In this way, $10-15 \mathrm{~mL}$ of platelet-rich plasma suspension was obtained (platelet count exceeding $800 \times 10^{9} / \mathrm{L}$ ). This was activated at the time of surgery with a $10 \%$ calcium chloride solution and $300 \mathrm{IU}$ of bovine thrombin (Fibriquick; Organon Teknika, Boxtel, The Netherlands) to form a gel, ${ }^{15,16}$ while the erythrocytes were given back. ${ }^{17,18}$

The PDLLA trays were fixed with the two preshaped osteosynthesis plates (Fig. 1). The soft tissues were closed in layers, using resorbable Vicryl 3-0 sutures. Immediate postoperative radiographs were made. Flunixin (Finadyne, $1.5 \mathrm{mg} / \mathrm{kg}$; Schering-Plough, Segre, France) was given for 3 days to alleviate postoperative pain. The procedure was performed unilaterally. After 6 weeks, the animals were killed by an overdose of pentobarbital.

\section{Radiographic evaluation}

Radiographs of the reconstructed sites were made directly after surgery. The mandibles were retrieved for radiographic and histological evaluation. Radiographs were made with a mobile X-ray machine (Mobilett B; Siemens, Erlangen, Germany), using a long-cone technique from a distance of $70 \mathrm{~cm}(40 \mathrm{kV}, 28 \mathrm{~mA})$. Two independent examiners assessed the radiographs from this experimental group. They were asked to score on a checklist as depicted in Table 1. Parameters involved included bridging of the defect by bone, callus formation at the lower borders of the defects, and resorption both at the lower border as well as centrally in the graft. The details of the scoring system are presented in Table $1 .{ }^{19}$

\section{Histological preparation}

Mandibular segments of each goat, including the grafted area, were fixed in a $4 \%$ phosphate-buffered formaldehyde solution ( $\mathrm{pH}$ 7.4). The segments were divided into four parts (parts A-D) analogous to previous experiments (Fig. 1). These parts were then dehydrated in graded series of alcohol and embedded in methylmethacrylate. Only part C was used in this study, as it was thought to be the part that would be the most difficult to revascularize. Three millimeters below the cut between parts B and C, a sagittal section plane was chosen.

Table 2. Parameters and Scoring System Used for Histologic Evaluation

\begin{tabular}{ll}
\hline Parameter & \multicolumn{1}{c}{ Score } \\
\hline Callus formation & $3=$ Complete across the defect \\
2 & $=$ Incomplete: covering $>50 \%$ of the defect \\
1 & $=$ Incomplete: covering $<50 \%$ of the defect \\
0 & $=$ None \\
& \\
Fibrous encapsulation & $0=$ Present \\
1 & $=$ None \\
\hline
\end{tabular}




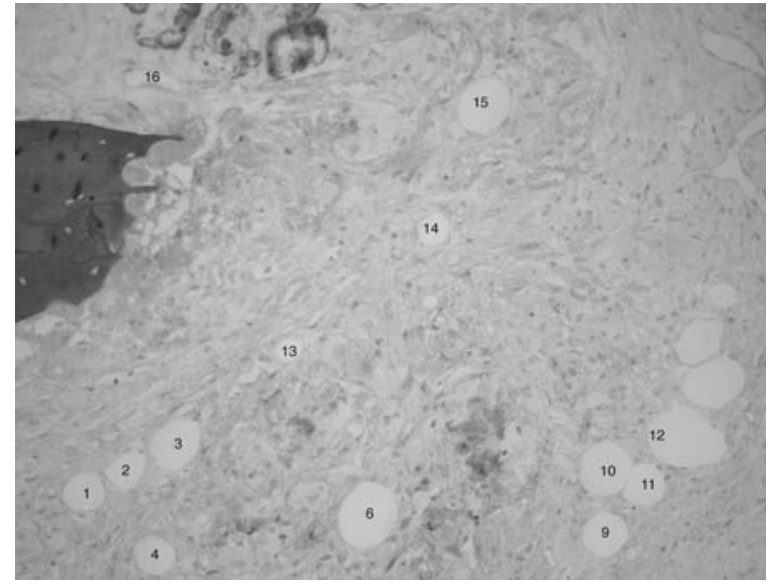

FIG. 2. Capillary count. Endothelial lining was paramount for identification of capillaries. (Fuchsin; original magnification, $\times 20$.)

After polymerization of each specimen, three $10-\mu \mathrm{m}$ transverse sections were made, using a modified microtome technique. ${ }^{20}$ Light microscopy sections were etched with hydrochloric ethanol and subsequently stained with methylene blue and basic fuchsin.

\section{Histological and histomorphometric evaluation}

Light microscopy analysis consisted of a description of the tissue response within the grafted area as well as the surrounding tissues.

Each specimen was quantitatively assessed on the basis of a grading scale for fibrous encapsulation and callus formation (Table 2).${ }^{19}$ For this purpose a microscopy image analysis system (Qwin Pro-image; Leica Microsystems, Wetzlar, Germany) was used. The sections were digitized at low magnification.

In each of the sections an area of interest was also defined, most centrally within the graft. In this area, after digitizing at high magnification, morphometric measurements were made in order to quantify capillary formation in the central area of the graft. Capillaries were identified by the presence of endothelial cells and erythrocytes. The number of capillaries per field was counted in three microscopic fields per section (Fig. 2).

\section{RESULTS}

All goats in the current experiment had an uneventful recovery and were walking within $3 \mathrm{~h}$. Within $36 \mathrm{~h}$, all six goats were eating and ruminating again. Their weight remained stable throughout the experiment.

On sacrifice no plate fractures were noted. In one animal three loose screws were seen. The five remaining specimens showed the same overall picture in that bone formation was seen partially overgrowing the PDLLA scaffolds as well as the apparently intact plates (Fig. 3). In all cases the defect macroscopically appeared to be bridged by newly formed bone on the buccal and lingual aspects of the mandible.

The radiographs taken at sacrifice confirmed three loose screws in one animal, all on the proximal side of the upper plate. No dislocation of the plate or mandibular segments was noted. None of the osteosynthesis plates showed any sign of fractures. In the defect zones, in five of six animals the bone graft seemed to have taken well; in one animal there seemed to be no continuity of bone. This animal showed severe resorption with only a narrow zone of continuous bone on the cranial side of the defect (Fig. 4). Callus formation at the lower border was seen in four of six animals. Five of six animals showed signs of resorption at the mandibular angle and lower border. The results of the scoring of radiographic parameters are presented in Table 3.

After making the cross-section for microscopic evaluation, macroscopic evaluation of the mandibular segments showed that the PDLLA scaffolds had shrunk, thus narrowing the space containing the grafted bone (Fig. 5). Light microscopy evaluation showed that extensive resorption of the particulate bone grafts had taken place, which was manifested by signs of osteoclastic activity on the few remaining bone chips within the confines of the PDLLA tray. The remaining space was occupied by mature fibrous tissue without any signs of inflammation. On the external surface of the PDLLA scaffolds, however, callus formation provided bone continuity between the mandibular stumps (Fig. 6). A considerable amount of callus formation was seen in four of six goats, covering most of the PDLLA scaffold. In two goats, however, the callus only partially covered the PDLLA scaffold. The remaining particulate bone chips showed signs of re-

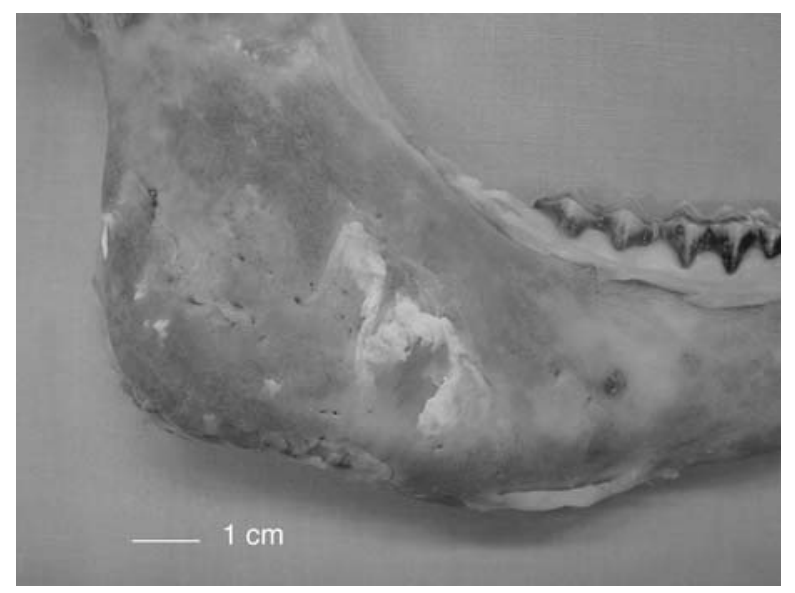

FIG. 3. Overgrowth of bone covers the osteosynthesis plates on the buccal aspect of the mandible and provides bony continuity. 


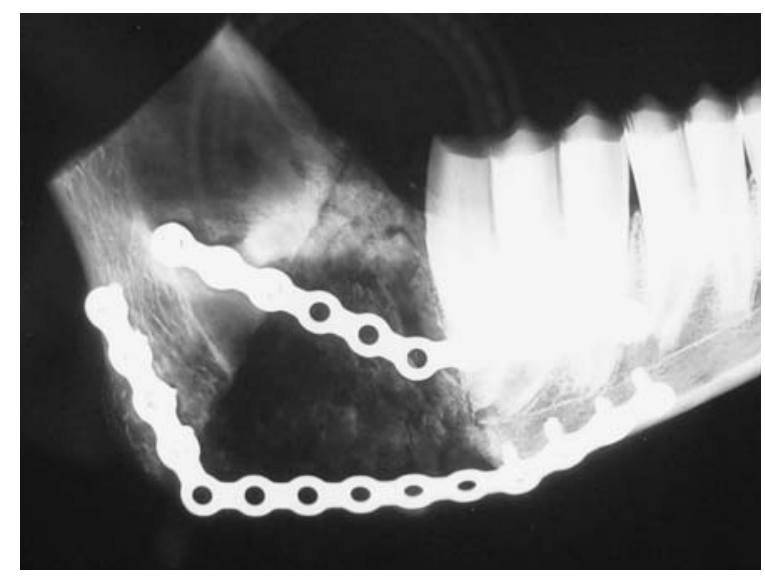

FIG. 4. Radiograph at 6 weeks showing extensive bone resorption and minimal bone continuity at the cranial rim.

modeling, predominantly osteoclastic activity and some osteoid tissue (Fig. 7). In the animal with the loose screws a necrotic grafted area without any signs of remodeling was seen, directly adjacent to the PDLLA scaffold. Yet, abundant callus formation had appeared, providing bony continuity of both mandibular stumps.

All specimens showed fibrous encapsulation of the PDLLA scaffold. In five of six animals, minimal signs of inflammation were seen in the form of one layer of inflammatory cells directly adjacent to the PDLLA. The PDLLA itself showed no signs of degradation. In the specimen with necrosis of the grafted bone, a more extensive fibrotic capsule with lymphocytic infiltration was seen.

Abundant bone apposition at the muscle attachment zones extending beyond the original contour of the mandible, as seen in previous experiments in which the original cortical bone tray was used, ${ }^{4,6}$ did not occur in the current experiment. The results of the quantitative histological scoring of fibrous encapsulation, callus formation, and capillary count are listed in Table 4.

\section{DISCUSSION}

It was the aim of this animal experiment to test the versatility of a technique in which the resected part of the

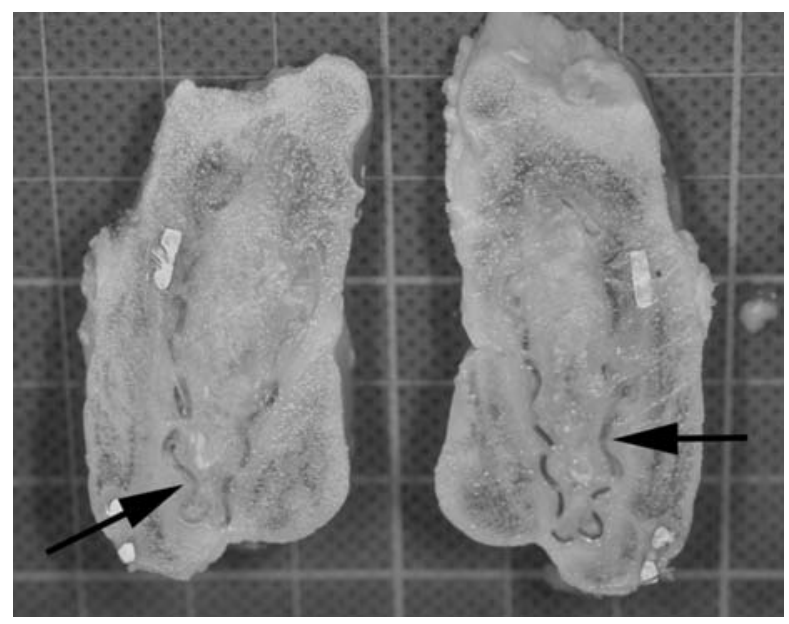

FIG. 5. Narrowing of the PDLLA scaffolds (arrows).

mandible was replaced by a resorbable poly(D,L-lactide) (PDLLA) scaffold, filled with an autogenous particulate cancellous bone graft from the anterior iliac crest, mixed with autogenous PRP. The hypothesis was that these grafts would heal, providing bone continuity with the grafted bone, within a period of 6 weeks. In all goats callus formation along the reconstructed segment was seen, providing bony continuity and maintaining the original contour of the reconstructed segment. The particulate bone grafts within the PDLLA scaffold, which appeared to be narrowed, showed considerable resorption and replacement by fibrous tissue. The model used in the current and in previous experiments ${ }^{3,4,6}$ has proven to allow for undisturbed oral function, including eating and ruminating throughout the experimental periods. This can be attributed to the stability provided by the preshaped $2.3-\mathrm{mm}$ plates and the monocortical screws. The three loose screws in one animal were all in the region of the ascending ramus, at the site where the thick neurovascular bundle enters the mandible. In previous experiments this was also seen in one animal, but only with one screw at the same site. The bone in this region is this, only 0.5 to $1.0 \mathrm{~mm}$; ideally, the posterior part of the cranial plate should have been placed at the anterior border of the ascending ramus. However, we supposed that this would be more liable to cause mucosal dehiscence. Oral function and clinical outcome, despite the loose screws, remained unchallenged.

Table 3. Mean Results of Radiological Scoring ${ }^{a}$

\begin{tabular}{lcccc}
\hline & Bone union & $\begin{array}{c}\text { Callus } \\
\text { formation }\end{array}$ & $\begin{array}{c}\text { External } \\
\text { resorption }\end{array}$ & $\begin{array}{c}\text { Central } \\
\text { resorption }\end{array}$ \\
\hline Mean & 1.5 & 0.583 & 0.833 & 0.5 \\
SD & 1.216 & 0.504 & 0.389 & 0.522 \\
\hline
\end{tabular}

${ }^{a} n=6,6$ weeks. Forms completed by two independent referees. 


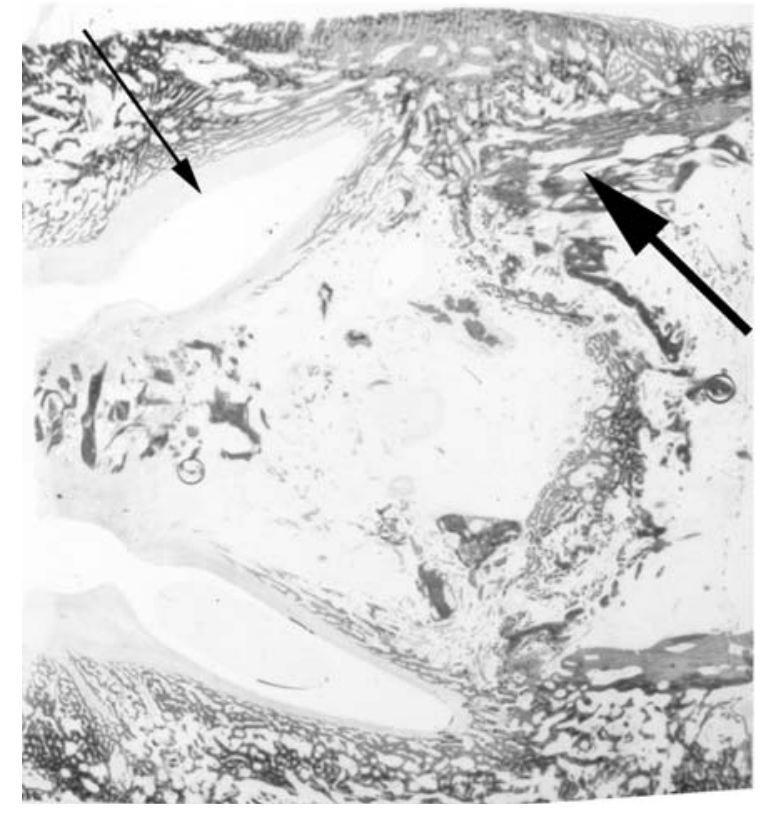

FIG. 6. Typical example of histology after 6 weeks. Collapse of the PDLLA scaffold with resorption of particulate bone graft (thin arrow). Callus provides continuity of bone with cortical bone stumps (thick arrow). (Fuchsin; original magnification, $\times 20$.)

In all animals, on sacrifice, the contour of the reconstructed mandible was similar to the original contour, seemingly indicating that the use of a PDLLA tray had maintained the shape.

Radiological evaluation indicated an absence of bony continuity in one of the six reconstructed mandibles. This, however, may be due to erroneous judgment as the PDLLA tray is completely radiolucent, in contrast to the cortical bone trays, which are more radio-opaque than the particulate cancellous bone grafts used. Because of this, there was variance in exposure times and kilovoltage. This led to high interobserver variances, especially when bone density was minimal. Thus, in those situations it was difficult to agree on radiographic continuity of bone. Computed tomography scanning may overcome this problem, for with computed tomography contrast ratios can be standardized and threshold values can be defined. Tomography also has the additional value of providing three-dimensional images, thus decreasing the chance of a false-positive outcome for bony continuity. Because of the differences in radiolucency of cortical bone and PDLLA, statistical comparison of the current group with previous groups was deemed unreliable.

The narrowing of the PDLLA tray as seen in the current experiment is probably due to the fast onset of degradation. The trays were manufactured by heat-molding a 0.5-mm PDLLA sheet around a template. During this process, the PDLLA sheet is stretched and, thus, becomes thinner. As soon as hydrolysis sets in, the mechanical strength decreases, with a rate depending on the crystallinity and molecular weight of the polylactide used. Thus, micromovements will occur because of the pressure of the soft tissue envelope surrounding the PDLLA scaffold. This may be one of the reasons why the particulate bone graft within the scaffold did not take. Kinoshita et al. described an experiment with mandibular continuity defects in dogs. ${ }^{21}$ In this experiment a polylactide mesh tray, filled with a particulate bone graft, was used. This mesh tray was made from 0.5 -mm PLA filaments, fixed to the mandibular stumps with circumferential steel wires; no collapse of this tray was seen. Schwartz et al., who used Dacron-urethane trays, reported on a similar experiment in dogs. ${ }^{22}$ These trays did not collapse either.

Bone formation on the outer aspect of the tray was seen in all animals in the current experiment, on the lingual side as well as on the buccal side, with the exception of two animals, in which minimal callus formation was seen on the lingual side. On the buccal side, the periosteum was removed or severely damaged, whereas on the lingual side, the periosteum was damaged but not removed so as to preserve the function of the lingual nerve. Schwartz et al., in their experiment using a Dacron-urethane mesh, described bone formation around the scaffold and in continuity with the bone within the scaffolds after 6 and 12 months, when the periosteum was left in place. ${ }^{22}$ After excision of the periosteum, no bone was seen outside the scaffold. Kinoshita et al. came up with similar results using a PLA mesh tray. ${ }^{21}$ We suppose that if bone formation, in the current experiment, on the outside of the scaffold would be of periosteal origin, this would predominantly occur on the lingual side.

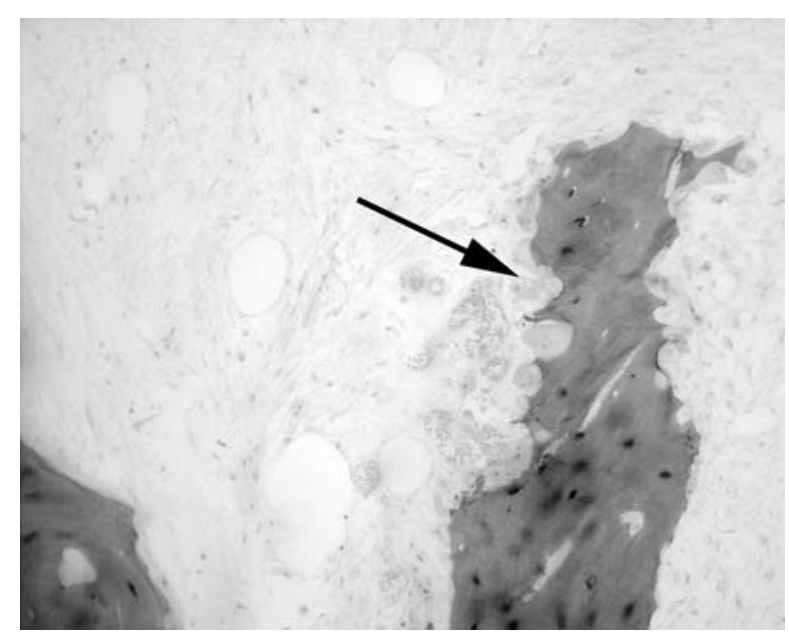

FIG. 7. Mature fibrous tissue within grafted area with osteoclastic activity on bone particles (arrow). (Fuchsin; original magnification, $\times 20$.) 
Table 4. Mean Results of Quantitative Histological Scoring

\begin{tabular}{lccc}
\hline & $\begin{array}{c}\text { Fibrous } \\
\text { encapsulation }\end{array}$ & $\begin{array}{c}\text { Callus } \\
\text { formation }\end{array}$ & $\begin{array}{c}\text { Capillary } \\
\text { count }\end{array}$ \\
\hline Mean & 1 & $2.5^{\mathrm{a}}$ & $14.72^{\mathrm{a}}$ \\
SD & 0 & 0.786 & 8.351 \\
\hline
\end{tabular}

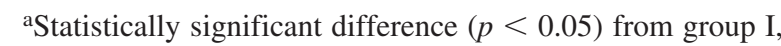
II, or III.

Furthermore, in goats, after periosteal elevation, the bone-lining cells remain on the cortical bone. In the current experiment, therefore, the lining cells cannot play a role of importance..$^{23}$ Possibly, the callus formation seen in the current experiment is due to migration of (pre)osteoblasts and growth factors released from the PRP gel through the perforations in the PDLLA tray. As the current experiment lacks a control group without the use of PRP gel, no definitive conclusion can be drawn. Comparison of callus formation in the present study with callus formation in our previous studies ${ }^{4,6}$ showed that no statistically significant differences exist between the current experimental group and all other previous treatment groups (Kruskal-Wallis test, Dunn's multiple comparison test, $p>0.05)$. Material and methods in these studies were standardized, with the exception of the scaffold used. In the current experiment, callus formation seems to have compensated for the compression of the scaffolds, leading to an anatomical contour of the reconstructed segment. The fact that no excessive bone formation was seen in the muscle attachment zones may be attributed to the fact that apposition of bone in the current experiment took place on the compressed scaffold instead of on the external surface of the uncompressed cortical bone trays. Another reason may be the osteoinductive properties of the cortical bone trays. Because of the compression of the PDLLA tray, it was not possible to select a reproducible and representative region of interest. Therefore, bone-fill percentage was not assessed in the current experiment.

Replacement of bone graft within the PDLLA scaffold by fibrous tissue probably reflects the rapid proliferation of fibroblasts into the scaffold at the expense of the proliferation of osteoblasts. Both effects can be attributed to growth factors released from PRP. ${ }^{24}$ The lack of bone formation directly adjacent to the PDLLA scaffold confirms the lack of osteoconductive capacity of PDLLA as compared with cortical bone in previous experiments. This may in part explain the relatively poor formation of new bone within the scaffold, despite the good revascularization of the graft as expressed by the high capillary count. Comparison of the capillary count data with the data of our previous studies shows that the capillary count in the current experimental group (PDLLA) is comparable to that of the group treated with cortical bone tray scaffolds filled with autogenous bone mixed with PRP (PRP). On the other hand, the capillary count in the current study was significantly higher compared with the group treated with cortical bone tray scaffolds filled with autogenous bone without PRP (Control) and the group treated with irradiated cortical bone tray scaffolds filled with autogenous bone mixed with PRP scaffolds (PRP + Irrad.) (Kruskal-Wallis test, Dunn's multiple comparison test, $p<0.01$ and $p<0.05$, respectively) (Fig. 8). This confirms the feasibility of replacing a cortical tray with a degradable PDLLA scaffold, but also confirms again

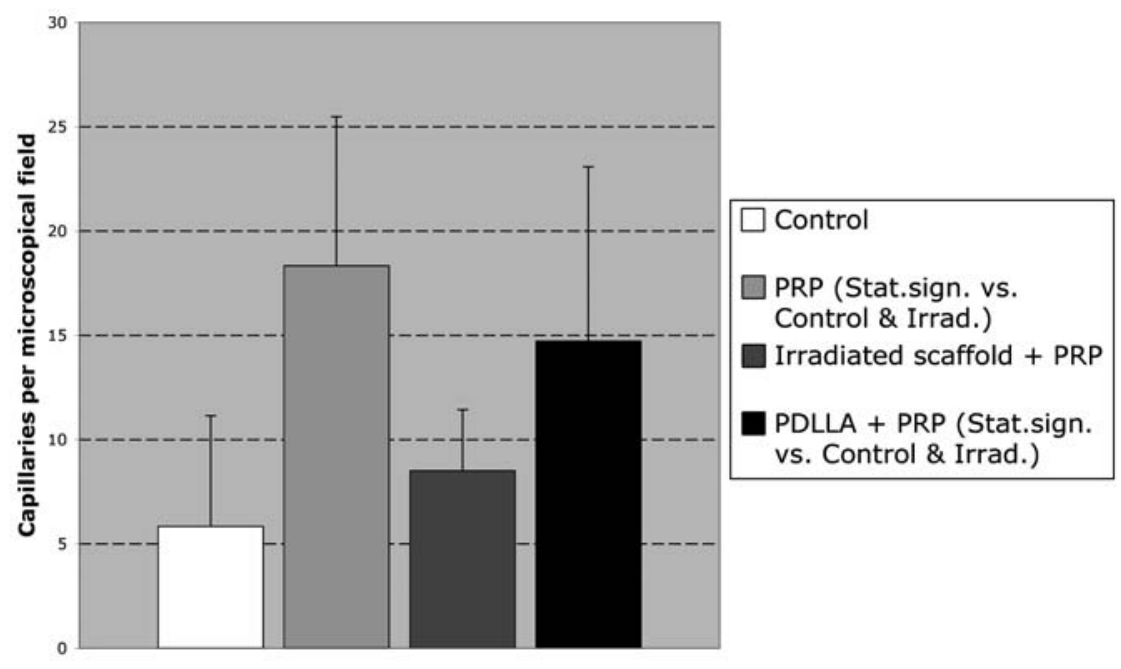

FIG. 8. Mean capillary count at 6 weeks in the current group (PDLLA) and in the PRP group was significantly higher ( $p<$ 0.01) than in the group without PRP or with irradiated scaffold. 
the effect of PRP on angiogenesis under physiological conditions.

\section{CONCLUSION}

On the basis of our observations, it is concluded that the results of the current study did not completely confirm the formulated hypothesis. The particulate bone graft within the PDLLA scaffold, which had been mixed with PRP, was found to be almost completely resorbed. Nevertheless, the clinical result was favorable as bony continuity between the mandibular stumps was provided by the newly formed bone on the outside of the scaffold, maintaining the original shape of the reconstructed area. The high rate of vascularization of the grafted area may also be attributed to PRP. In view of the results presented, a PDLLA scaffold appears to form a promising alternative to maintain the original contour in mandibular reconstruction.

\section{ACKNOWLEDGMENTS}

This study was supported by Martin Medizintechnik (Tüttlingen, Germany) and the Research Foundation of the Dutch Association of Oral and Maxillofacial Surgery. The authors thank Dr. Petra de Pooter for help in evaluating the radiographs.

\section{REFERENCES}

1. Rogers, S.N., Lakshmiah, S.R., Narayan, B., et al. A comparison of the long-term morbidity following deep circumflex iliac and fibula free flaps for reconstruction following head and neck cancer. Plast. Reconstr. Surg. 112, 1517, 2003.

2. Hartman, E.H., Spauwen, P.H., and Jansen, J.A. Donor-site complications in vascularized bone flap surgery. J. Invest. Surg. 15, 185, 2002.

3. Fennis, J.P., Stoelinga, P.J., and Jansen, J.A. Mandibular reconstruction: A clinical and radiographic animal study on the use of autogenous scaffolds and platelet-rich plasma. Int. J. Oral Maxillofac. Surg. 31, 281, 2002.

4. Fennis, J.P., Stoelinga, P.J., and Jansen, J.A. Mandibular reconstruction: A histological and histomorphometric study on the use of autogenous scaffolds, particulate cortico-cancellous bone grafts and platelet rich plasma in goats. Int. J. Oral Maxillofac. Surg. 33, 48, 2004.

5. Jisander, S., Aspenberg, P., Salemark, L., and Wennerberg, J. Mandibular reconstruction by secondary reimplantation of resected segments: A preliminary report. Int. J. Oral Maxillofac. Surg. 24, 288, 1995.

6. Fennis, J.P.M., Stoelinga, P.J.W., and Jansen, J.A. Reconstruction of the mandible with an autogenous irradiated cortical scaffold, autogenous corticocancellous bone-graft and autogenous platelet-rich-plasma: An animal experiment. Int. J. Oral Maxillofac. Surg. 34, 158, 2005.

7. Cheung, L.K., Samman, N., Tong, A.C., and Tideman, H. Mandibular reconstruction with the Dacron urethane tray: A radiologic assessment of bone remodeling. J. Oral Maxillofac. Surg. 52, 373, 1994.

8. Dumbach, J., Rodemer, H., Spitzer, W.J., and Steinhauser, E.W. Mandibular reconstruction with cancellous bone, hydroxylapatite and titanium mesh. J. Craniomaxillofac. Surg. 22, 151, 1994.

9. Eckardt, A., and Kahre, D. Polyurethane-coated Dacron mesh tray for temporary mandibular reconstruction following resection of oral cancer: Clinical results of 27 cases. J. Oral Maxillofac. Surg. 47, 21, 1989.

10. Leake, D.L. A new alloplastic tray for osseous contour defects. J. Maxillofac. Surg. 2, 146, 1974.

11. Leake, D.L. Mandibular reconstruction with a new type of alloplastic tray: A preliminary report. J. Oral Surg. 32, 23, 1974.

12. Tideman, H., Samman, N., and Cheung, L.K. Functional reconstruction of the mandible: A modified titanium mesh system. Int. J. Oral Maxillofac. Surg. 27, 339, 1998.

13. Merkx, M.A., Maltha, J.C., Freihofer, H.P., and KuijpersJagtman, A.M. Incorporation of three types of bone block implants in the facial skeleton. Biomaterials 20, 639, 1999.

14. Clemmons, R.M., Bliss, E.L., Dorsey-Lee, M.R., Seachord, C.L., and Meyers, K.M. Platelet function, size and yield in whole blood and in platelet-rich plasma prepared using differing centrifugation force and time in domestic and food-producing animals. Thromb. Haemost. 50, 838, 1983.

15. Tayapongsak, P., O’Brien, D.A., Monteiro, C.B., and Arceo-Diaz, L.Y. Autologous fibrin adhesive in mandibular reconstruction with particulate cancellous bone and marrow. J. Oral Maxillofac. Surg. 52, 161, 1994.

16. Whitman, D.H., Berry, R.L., and Green, D.M. Platelet gel: An autologous alternative to fibrin glue with applications in oral and maxillofacial surgery. J. Oral Maxillofac. Surg. 55, 1294, 1997.

17. Ledent, E., Wasteson, A., and Berlin, G. Growth factor release during preparation and storage of platelet concentrates. Vox Sang. 68, 205, 1995.

18. Marx, R.E., Carlson, E.R., Eichstaedt, R.M., Schimmele, S.R., Strauss, J.E., and Georgeff, K.R. Platelet-rich plasma: Growth factor enhancement for bone grafts. Oral Surg. Oral Med. Oral Pathol. Oral Radiol. Endod. 85, 638, 1998.

19. An, Y.H., and Friedman, R.J. Animal Models in Orthopedic Research. Boca Raton, FL: CRC Press, 1999, p. 251.

20. van der Lubbe, H.B., Klein, C.P., and de Groot, K. A simple method for preparing thin $(10 \mu \mathrm{m})$ histological sections of undecalcified plastic embedded bone with implants. Stain Technol. 63, 171, 1988.

21. Kinoshita, Y., Kobayashi, M., Hidaka, T., and Ikada, Y. Reconstruction of mandibular continuity defects in dogs using poly(L-lactide) mesh and autogenic particulate cancellous bone and marrow: Preliminary report. J. Oral Maxillofac. Surg. 55, 718, 1997. 
22. Schwartz, H.C., Leake, D.L., and Pizzoferrato, A. Evaluation of autologous cancellous bone grafting of mandibular discontinuity defects in dogs using a Dacron-urethane prosthesis. J. Oral Maxillofac. Surg. 45, 143, 1987.

23. O'Driscoll, S.W., Recklies, A.D., and Poole, A.R. Chondrogenesis in periosteal explants: An organ culture model for in vitro study. J. Bone Joint Surg. Am. 76, 1042, 1994.

24. Schliephake, H. Bone growth factors in maxillofacial skeletal reconstruction. Int. J. Oral Maxillofac. Surg. 31, 469, 2002.
Address reprint requests to: J.P.M. Fennis, D.D.S., M.D., Ph.D. Department of Oral and Maxillofacial Surgery Rijnstate Hospital Wagnerlaan 55, 6815 AD Arnhem, The Netherlands

E-mail: j.fennis@chello.nl 